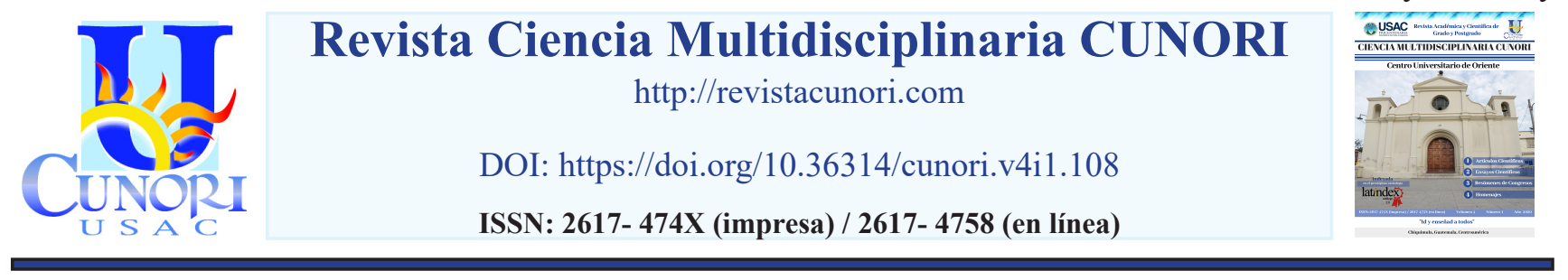

Como citar el artículo

Morales, J. (2020). Utilidad y aplicaciones de las tecnologías convergentes. Revista Ciencia Multidisciplinaria CUNORI, 4(1), 43-53. DOI: https://doi.org/10.36314/cunori.v4i1.108

\title{
Utilidad y aplicaciones de las tecnologías convergentes
}

\section{Utility and applications of convergent technologies}

\author{
Jorge Estuardo Morales \\ Departamento de Actualización para Adultos, Universidad Galileo, Doctorado en Administración de Empresas y Capital Humano \\ Recibido: 16 de marzo de 2019 / Revisión: 30 de abril de 2019 / Aceptado: 12 de febrero de 2020 \\ Disponible en internet el 29 de mayo de 2020 \\ *Autor para correspondencia. \\ Correo electrónico: jmorales.0807@gmail.com
}

Resumen

- $\mathrm{s}$ evidente que en la actualidad se vive una rápida evolución de la tecnología y de la ciencia, dándose una transición para que

Clas mismas pasen a convertirse como los factores productivos primordiales dentro del mundo moderno, para lo cual el conocimiento continúa siendo un pilar fundamental. Hoy en día, el avance en el conocimiento ya no sucede en forma acumulativa, sino más bien ocurre a través de la ciencia como síntesis, es decir, con un enfoque de integración en lugar de especialización, con diálogo y aprendizaje recíproco en lugar de transmisión pasiva. La integración y aprendizaje recíproco son ingredientes fundamentales de las ciencias y tecnologías convergentes las cuales son conocidas con el acrónimo NBIC que básicamente sintetiza la integración, interdisciplinariedad y multidisciplinariedad del conocimiento científico, cada letra del acrónimo corresponde a una disciplina: nano: nanociencia y nanotecnología; bio: biotecnología; info: tecnología de la ciencia de la información; y cogno: ciencias cognitivas o conocimiento tecnocientífico. La competitividad y la búsqueda de respuestas y soluciones a problemas sociales, ambientales y de salud, constituyen factores que motivan la convergencia e integración de tecnologías. Cada día se irán desarrollando más aplicaciones de las NBIC en diversos campos como la industria, la salud, la informática, energía, ambiente, problemáticas sociales, entre otros, caracterizándose principalmente porque la ciencia no se define a partir de la especialización, sino más bien por la interdisciplinariedad de conocimientos en procura de la solución de problemas en común.

Palabras clave: ciencia como síntesis, tecnociencia, genoma humano, proteómica, fullerenos

Abstract

$I^{1}$ $\mathrm{t}$ is evident that today there is a rapid evolution of technology and science, giving a transition so that they become the primary production factors in the modern world, for which knowledge continues to be a fundamental pillar. Today, advancement in knowledge no longer happens cumulatively, but rather occurs through science as a synthesis, that is, with an approach of integration rather than specialization, with dialogue and reciprocal learning instead of transmission. passive. Integration and reciprocal learning are fundamental ingredients of the converging sciences and technologies which are known with the acronym NBIC that basically synthesizes the integration, interdisciplinarity and multidisciplinarity of scientific knowledge, each letter of the acronym corresponds to a discipline: nano: nanoscience and nanotechnology; bio: biotechnology; info: information science technology; and cogno: cognitive sciences or technoscientific knowledge. Competitiveness and the search for answers and solutions to social, environmental and health problems are factors that motivate the convergence and integration of technologies. Every day more applications of NBICs will be developed in various fields such as industry, health, computing, energy, environment, social problems, among others, characterized mainly because science is not defined by specialization, but rather for the interdisciplinarity of knowledge in search of the solution of common problems.

Keywords: science as synthesis, technoscience, human genome, proteomics, fullerenes 


\section{Introducción}

El desarrollo de la ciencia y la tecnología se distingue en la actualidad por los cambios acelerados que exigen capacidad de respuesta a las exigencias del entorno en términos de competitividad y de los problemas que afectan a las sociedades en diferentes ámbitos como salud, ambiente, empresarial, economía, entre otros; para lo cual la creación de conocimiento basado en redes de trabajo de investigación y colaboración constituye la principal característica de las tecnologías convergentes.

El propósito fundamental del presente documento consiste identificar las principales aplicaciones de las ciencias y tecnologías convergentes y los beneficios que conllevan, así como establecer los factores que favorecen la tendencia de su utilización; para lo cual se aplicó la estrategia de investigación documental.

Mediante el desarrollo de la temática es posible brindar claridad en los conceptos asociados a las tecnologías convergentes, su evolución y tendencias, los beneficios que conlleva su aplicación en distintas áreas y en consecuencia, comprender la importancia de la integración científica entre la tecnología y la ciencia en diversas disciplinas que se apoyan para el eficiente diseño y creación de artefactos tecnológicos y soluciones creativas a problemas que afectan a la sociedad. A través de dicha comprensión, se logra el compromiso e interés de las personas para involucrarse directa o indirectamente en el desarrollo de las tecnociencias en mención.

El objetivo principal de la convergencia de ciencias y tecnologías consiste en lograr la transferencia del conocimiento científico para generar innovación para la sociedad, favoreciendo la generación de riqueza y de desarrollo económico de los países. La idea básica consiste en conformar un sistema científico integrado que genere sinergia en la búsqueda de aplicaciones y soluciones creativas en diversos ámbitos. Un ejemplo se observa a través del conocimiento organizacional, en donde el desarrollo y mejora de la creatividad y la innovación, permite el logro de los niveles de competitividad deseados; o bien, el conocimiento científico para resolver problemas de salud, producción, manufactura y otros diversos.

El contenido del ensayo está estructurado inicialmente con la descripción del proceso de transformación de la ciencia y la tecnología. Luego se identifican y describen las disciplinas que integran las ciencias y tecnologías convergentes; seguidamente se explican sus funciones y aplicaciones; continuando el desarrollo con la ilustración y explicación de los factores que impulsan la convergencia de las ciencias y tecnologías; subsiguientemente, se presentan ejemplos de aplicaciones y beneficios de las ciencias y tecnologías de síntesis. Finalmente, se presentan las conclusiones de la temática expuesta, mismas que responden las inquietudes y propósitos establecidos.

\section{Proceso de transformación en la ciencia y tecnología}

La importancia que ha tenido el conocimiento en diferentes momentos de la historia, no siempre ha tenido la misma proporción. En la sociedad agrícola el trabajo como factor de producción era considerado como el más importante, en la sociedad industrial fue el factor capital el más relevante, luego en la sociedad de la información fue ésta última la de mayor jerarquía y posteriormente, en la sociedad del conocimiento, 
la información sumada al aprendizaje se convierte en conocimiento, siendo este último el factor más importante; es decir que el conocimiento es la base para el desarrollo de la sociedad y la información continúa siendo necesaria, mientras que el capital y el trabajo ya no son los factores más relevantes.

El acelerado desarrollo de la ciencia y de la tecnología como fuerzas productivas principales, revela el destacado papel transformador del conocimiento, donde la transformación cualitativa es más significativa que la acumulación cuantitativa del conocimiento, siendo la base para la apertura a nuevas perspectivas de desarrollo social y económico. (Sáenz, 2006). De la afirmación anterior, cabe el siguiente cuestionamiento, ¿en realidad, la ciencia y la tecnología se están convirtiendo en los elementos más importantes para el avance y progreso de la sociedad?

Es evidente que en la actualidad se vive una rápida evolución de la tecnología y de la ciencia, dándose una transición para que las mismas pasen a convertirse como los factores productivos primordiales dentro del mundo moderno, para lo cual el conocimiento continúa siendo un pilar fundamental. Hoy en día, el avance en el conocimiento ya no sucede en forma acumulativa, sino más bien ocurre a través de la ciencia como síntesis, es decir, con un enfoque de integración en lugar de especialización, con diálogo y aprendizaje recíproco en lugar de transmisión pasiva.

Cada día que transcurre, se da un proceso cada vez más acelerado de actualización y mejora tecnológica que tiene impactos significativos de transformaciones no sólo en el ámbito de la producción, sino además en la generación y aplicación del conocimiento para poder encontrar soluciones a los diversos problemas que se presenten. Consecuentemente, el trabajo autónomo y creativo adquiere gran importancia dado que las personas ya no se especializan únicamente en ejecutar funciones rutinarias sino que deben estar calificadas para desempeñarse en un amplio conjunto de funciones y a la vez, es indispensable su participación en actividades de aprendizaje colaborativo en pro del desarrollo del conocimiento y de la ciencia para beneficio de la sociedad.

La integración y aprendizaje recíproco son ingredientes fundamentales de las ciencias y tecnologías convergentes, pero ¿qué son estas ciencias y tecnologías? Las ciencias y tecnologías convergentes son conocidas con el acrónimo NBIC, las cuales se identifican y describen en la tabla 1. NBIC básicamente sintetiza la integración, interdisciplinariedad y multidisciplinariedad del conocimiento científico, cada letra del acrónimo corresponde a una disciplina: nano: nanociencia y nanotecnología; bio: biotecnología; info: tecnología de la ciencia de la información; y cogno: ciencias cognitivas o conocimiento tecnocientífico; expresando así la unión disciplinar. 
Tabla 1: Disciplinas que integran las ciencias y tecnologías convergentes

\begin{tabular}{|c|c|c|}
\hline & Disci & Descripción \\
\hline $\mathrm{N}:$ & $\begin{array}{l}\text { Nanociencia y } \\
\text { nanotecnología }\end{array}$ & $\begin{array}{l}\text { Conocimiento tecnocientífico de la materia orientado al } \\
\text { microanálisis de los materiales o de la manipulación de sus } \\
\text { componentes básicos a nivel atómico para lograr } \\
\text { estructuras moleculares con nuevas propiedades. Las } \\
\text { nanociencias y nanotecnologías se desarrollan y } \\
\text { coevolucionan sin límites, en combinación con la química } \\
\text { y la biología. }\end{array}$ \\
\hline B: & Biotecnología & $\begin{array}{l}\text { Conocimiento tecnocientífico que integra la bioquímica } \\
\text { con la ingeniería para lograr nueva producción molecular, } \\
\text { incorporando técnicas procedentes de la biología celular y } \\
\text { otras técnicas bioquímicas empleadas con fines tanto } \\
\text { científicos como industriales. La física y la bioquímica se } \\
\text { alían científicamente con estos planteamientos } \\
\text { nanobiotecnológicos. }\end{array}$ \\
\hline I: & $\begin{array}{l}\text { Tecnología y } \\
\text { ciencia de la } \\
\text { información }\end{array}$ & $\begin{array}{l}\text { El conocimiento de la computación moderna y de las } \\
\text { comunicaciones avanzadas, basadas en los sistemas de } \\
\text { inteligencia artificial que procesan altos volúmenes de } \\
\text { datos en tiempos cortos, transformándolos en información } \\
\text { y conocimiento. }\end{array}$ \\
\hline C: & $\begin{array}{l}\text { Ciencias cognitivas } \\
\text { o conocimiento } \\
\text { tecnocientífico }\end{array}$ & $\begin{array}{l}\text { Conocimiento tecnocientífico que analiza el proceso de } \\
\text { creación del conocimiento humano y organizacional. En } \\
\text { esta categoría se relacionan ciencias y técnicas específicas, } \\
\text { tales como la psicología, lingüística, filosofía; } \\
\text { neurociencia, antropología, sociología y la disciplina de la } \\
\text { dirección o el gobierno del conocimiento organizativo. }\end{array}$ \\
\hline
\end{tabular}

Bueno (2006). Las ciencias y tecnologías convergentes (NBIC): análisis de su papel en los parques científicos como espacios y agentes de $\mathrm{I}+\mathrm{D}+\mathrm{i}$.

Las tecnologías convergentes conllevan avances rápidos a través de diversas áreas de la tecnología, donde los avances en un área aceleran el progreso en otras. En el futuro, en un mediano plazo, surgirán nuevos conocimientos científicos y tecnologías como resultado de la convergencia de la nanotecnología, la biotecnología, las tecnologías de información y cognoscitivas. Esta integración incidirá en la sinergia que se requiere para alcanzar elevadas mejorías en las habilidades humanas que impulsarán avances sociales. 


\section{Funciones y aplicaciones de las tecnologías convergentes}

Durante 2,500 años, la estructura de la educación clásica ha sido de especialización del conocimiento en ramas o carreras profesionales con un sentido egocentrista porque se llega a saber mucho de una materia a través de las especialidades pero muy poco de todo. Ahora la ciencia involucra integración y se caracteriza por la conformación de redes de trabajo y de cooperación para conformar grandes equipos de investigadores. El científico contemporáneo lleva a cabo un nuevo rol como experto en tecnociencia, que significa una convergencia e integración de disciplinas, unas como ciencias y otras como técnicas.

Es innegable la metamorfosis de la ciencia moderna con el avance de las ciencias y tecnologías convergentes que cumplen la función de alianza científica que implica la relación teórica y práctica de conocimientos, en un proceso interactivo distinguido por la diversidad de conceptos y sujetos de conocimiento partícipes en un proyecto de investigación común. Dicha convergencia, requiere de mucha investigación y desarrollo para dar sentido o explicación a las demandas actuales de la sociedad del conocimiento y de los agentes que la conforman. (Bueno, 2006).

Las tecnologías convergentes suponen la mayor revolución de todos los tiempos debido a que como explica Castilla, A., citado por Rodríguez (2009), permitirá a los individuos expandir sus habilidades de conocimiento y comunicación, aumentar sus capacidades físicas, mejorar su salud, aumentar las capacidades de entendimiento social, la seguridad y, por supuesto, mejorar la productividad y el crecimiento económico. En este nuevo contexto, la interrelación entre el orden económico capitalista y el poder político basan la dominación del mundo en los saberes científico-técnicos y sus beneficios económicos, sociales e individuales.

Surge la interrogante, ¿las tecnologías convergentes son fuentes de poder económico, político y social? "Las inversiones públicas y privadas en nanotecnología han crecido de manera sostenida desde el comienzo de este siglo. Las mercancías que incorporaron algún procedimiento nanotecnológico están llegando a los anaqueles de los comercios. A menudo se adjudica a las nanotecnologías un papel especial en el desarrollo; por un lado, las tecnologías eficientes y de bajo costo permitirían a buena parte de la población de los países en desarrollo, acceder al agua potable, a la energía solar y al diagnóstico y tratamiento de algunas enfermedades. Ello podría ser el punto de partida de un cambio radical en las condiciones de pobreza y un impulso al desarrollo" (Foladori e Invernizzi, 2008, pp.7-8)

Significa que a través de las NBIC es posible encontrar soluciones a problemas sociales, no obstante, requiere de la inversión de tiempo, esfuerzo, dinero, trabajo colaborativo, así como del interés y apoyo político de los Gobiernos a través de políticas de Estado y de sus Instituciones para promover proyectos basados en estas tecnociencias. Asimismo, se requiere del apoyo y trabajo conjunto del sector empresarial y organizaciones no gubernamentales para unificar esfuerzos en la búsqueda de soluciones a problemas que afectan a las sociedades. No es difícil de concebir que las tecnologías convergentes abren la posibilidad a descubrir nuevos conocimientos y, en definitiva, el saber es fuente de poder que puede servir de base para impulsar el comercio, la economía, las políticas gubernamentales, los proyectos sociales para atención y solución de problemas y necesidades, entre otros. 


\section{Factores impulsores de las tecnologías convergentes}

Existen diversos factores que impulsan el auge de los enfoques científico-tecnológicos de las NBIC, entre los cuales destacan: el tiempo y costo creciente en el desarrollo de medicamentos; la tendencia hacia la personalización de la medicina; la existencia de personal investigador entrenado en bioinformática, neuroinformática y nanomedicina; la madurez de la industria de dispositivos médicos; la existencia de diversos casos de éxito como los biochips; la existencia de una cultura de alianzas entre empresas y de investigación cooperativa en el entorno académico. (Martín-Sánchez y López-Campos, 2006). La figura 1, ilustra los factores mencionados.

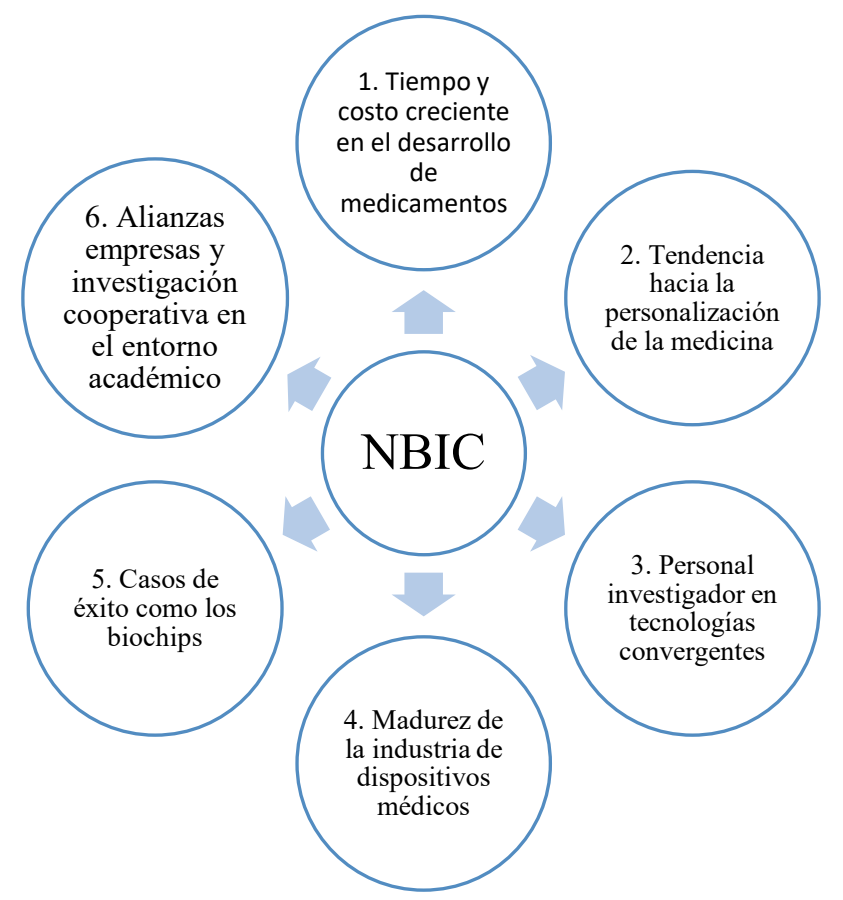

Figura 1: "Factores impulsores de las NBIC"

Martín-Sánchez y López-Campos (2006). Convergencia de Tecnologías: nuevas oportunidades para el avance de la informática y de las ciencias biomédicas.

El primer factor se refiere a la búsqueda de la industria farmacéutica de nuevos métodos de diseño y desarrollo de medicamentos más seguros y efectivos. El segundo impulsor está relacionado con el desarrollo de la genómica y de la terapia celular que ofrece la posibilidad de crear sistemas de diagnóstico y terapéuticos más adaptados a cada persona. El tercer elemento indica que en la actualidad los programas educativos y las instituciones de investigación han formado personas en áreas como la bioinformática, neuroinformática y nanomedicina.

El cuarto componente lo representan las empresas existentes con la capacidad de llevar a cabo diagnósticos basados en imagen molecular y funcional, así como en dispositivos analíticos miniaturizados. El quinto factor lo representan los biochips en los cuales convergen la biotecnología y la electrónica y que 
son utilizados para analizar procesos moleculares en células y tejidos. Finalmente, el sexto impulsor son las empresas que conforman alianzas para generar conocimiento e impulso de tecnologías, para lo cual crean sinergias en financiación y en la conformación de redes y centros virtuales de investigación.

Cabe analizar, si ¿existen otros factores impulsores de la convergencia de las NBIC?, básicamente sí, porque además de los seis factores identificados, existen otros elementos que estimulan las tecnologías convergentes, tal es el caso de los cambios y exigencias aceleradas del mundo contemporáneo; el progreso tecnológico; el comercio envuelto en un entorno altamente dinámico; así como los problemas consecuentes de dicho progreso y avance tecnológico. Como lo afirman Foladori e Invernizzi (2008), los beneficios de las nuevas tecnologías no han sido para todos ya que en las últimas décadas el fantástico ritmo de innovación tecnológica ha ido de la mano con el aumento de la desigualdad en el mundo.

Lo anterior, quiere decir que la necesidad de buscar la competitividad que exige el entorno mundial, es un elemento que impulsa la convergencia de tecnologías, sin embargo, también significa que la búsqueda de respuestas a problemas sociales, ambientales y de salud que han sido originados en muchos casos por los mismos avances y desarrollo de la humanidad, constituye otro factor que motiva la integración de ciencias y tecnologías. En el presente se vienen entrelazando puentes entre áreas de conocimiento porque se comparte el saber científico, donde la tendencia se dirige hacia las ciencias contemporáneas que se enfocan en problemas porque las personas se agrupan colaborativamente por las dificultades y necesidades que les afectan o interesan.

\section{Ejemplos de aplicaciones y beneficios de las NBIC}

Un primer ejemplo es la transversalidad de las ciencias de la computación en múltiples campos como la genómica y las guías genéticas diseñadas en laboratorio como el CRISP que es una nueva técnica para modificar el genoma humano con fines de corrección de genes en enfermedades hereditarias y de erradicar la enfermedad genética en su línea germinal. Un segundo ejemplo es la convergencia en la evolución de la microelectrónica y la nanofabricación, que han logrado la creación de sensores y dispositivos para aplicaciones biomédicas. (Casalet, 2017).

El proyecto genoma humano se divide en dos fases, la primera fase consiste en leer el mapa genético o identificar genes, por ejemplo, predecir si en una familia hay propensión a diabetes, padecimientos cardíacos u otros. La segunda etapa es la proteómica que significa escribir sobre el mapa genético, por ejemplo, analizar qué pasa si se cambia un gen por otro. Al igual que el proyecto de genoma humano, han surgido una serie de programas de investigación grandiosos como la exploración del espacio exterior, la exploración del fondo submarino, la búsqueda de energías alternativas, entre otros.

Una ejemplificación más se encuentra en la manufactura aditiva o fábricas de escritorio que constituye un área que revoluciona las máquinas y el software, tal es el caso de la impresora 3D que juega un papel similar al de una impresora de documentos pero que puede crear objetos tridimensionales que se utilizan como componentes de productos o de medios de transporte, como el avión Airbus A350 que tiene múltiples piezas impresas con tecnología 3D, y de manera similar se utiliza en la industria automotriz. (Casalet, 2017). 
Las nanotecnologías implican productos con nanomateriales que juegan un papel fundamental en la alta eficiencia de las células solares y baterías, control ambiental con nanofiltros, o nanosistemas para aplicaciones médicas, electrónicas y dispositivos computacionales. El automóvil eléctrico ejemplifica cómo las nuevas baterías permiten aumentar la autonomía de los vehículos. El más reciente de los materiales con usos y aprovechamientos sorprendentes, es el fullereno.

Los fullerenos fueron descubiertos por Robert F. Curl, Sir Harold W. Kroto y Richard E. Smalley en 1985 .Once años después los tres científicos recibieron el Premio Nobel de Química del año 1996. Este descubrimiento ha dado lugar a un nuevo campo de estudio en diferentes ámbitos de la ciencia. Los fullerenos son moléculas estructuradas en forma de jaulas y están constituidos por una red formada por átomos de carbono en pentágonos y hexágonos, cerrando la esfera. La investigación exhaustiva sobre las aplicaciones biomédicas de esta molécula ha estado en marcha desde su descubrimiento, en la actualidad se sabe que los fullerenos son excelentes antioxidantes, tienen potencial como agentes antivirales, son fotosensibilizadores en terapias fotodinámicas, entre otros (Martín, 2011).

Los ejemplos de aplicaciones de las NBIC son variados y múltiples, no obstante, la figura 2, ilustra la relación entre las tecnologías convergentes y las áreas de aplicación donde la convergencia debe ser enfocada a la resolución de problemas de impacto en los distintos campos donde son utilizadas estas tecnociencias.

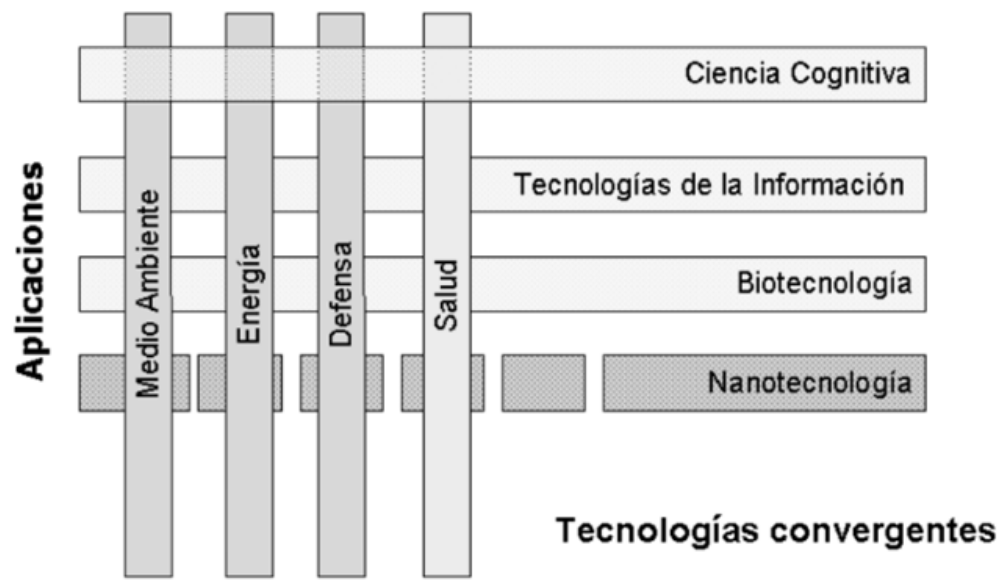

Figura 2: "Relación entre las tecnologías convergentes y sus áreas de aplicación

Martín-Sánchez y López-Campos (2006). “Convergencia de Tecnologías: nuevas oportunidades para el avance de la informática y de las ciencias biomédicas". p.4.

Un ejemplo destacable de la aplicación de la nanotecnología en el área de salud, es el caso de la científica guatemalteca Andrea Celeste del Valle quien logró crear una cura contra el cáncer terminal luego de ingresar y estudiar varios años en la National Tsing Hua University de Taiwán. El descubrimiento de la guatemalteca se basa en el principio que las células resistentes a todas las quimioterapias son paradójicamente muy sensibles a otro tipo de terapias. Con base en este conocimiento, la científica creó tres formas diferentes para matar el cáncer resistente, basándose en la nanotecnología y sus beneficios 
al acumularse selectivamente en el tumor, para lo cual creó partículas de hierro, oro y platino, las cuales utilizan imanes o láseres para destruir el tumor resistente a todo tipo de terapias de una manera sorprendente. (Mejía, 2019).

Cada día se irán encontrando y desarrollando más y más aplicaciones de las tecnologías convergentes en diversos campos como la industria, la salud, la informática, energía, ambiente, problemáticas sociales, entre otros. Una ejemplificación adicional se encuentra en la iniciativa BRAIN que es un acrónimo que significa: investigación del cerebro a través del avance de las tecnologías aplicadas a las neuronas, con el fin de comprender las funciones dinámicas del cerebro y demostrar aplicaciones de punta basadas en estos descubrimientos.

Otra posibilidad de aplicación se encuentra en la analítica de datos y los mecanismos necesarios de interacción que incluya principios y métodos de las estadísticas, matemáticas y ciencias cognitivas que facilite la manipulación y comprensión de datos masivos y dinámicos. Un fenómeno reciente es el Big Data o ciencia de los grandes datos, la cual se expresa a través de aplicaciones, analítica de la infraestructura, infraestructura operacional, infraestructura de servicios, bases de datos, analítica de la visualización, empresas inteligentes e implica grandes procesos de síntesis de conocimiento.

El progreso en la ciencia y tecnología ha evolucionado significativamente a partir de la década de los años sesenta hasta la actualidad, a partir de donde se han originado nuevas ciencias tales como las cognitivas, de la tierra, de la vida, de la salud, de los materiales, del espacio, de la complejidad, entre otras. La principal característica de esta evolución es que por primera vez, la ciencia no se define a partir del objeto o especialización, sino más bien por la interdisciplinariedad de conocimientos en procura de la solución de problemas en común.

Todo lo anterior representa la transición de la economía de la información a la economía del conocimiento, donde la división digital del mundo es un fenómeno cultural porque todos los registros operacionales y de toma de decisiones se encuentran en su mayoría alojados en espacios virtuales. Asimismo, la humanidad se encuentra en medio de una revolución científica por la convergencia de tecnologías y ciencias en pro del avance en el conocimiento que ya no sucede en forma acumulativa, sino a través de síntesis, es decir, de integración y colaboración.

\section{Conclusión}

Las principales aplicaciones de las ciencias y tecnologías convergentes están orientadas hacia un enfoque integrador que permite a las personas expandir sus habilidades de conocimiento y comunicación, aumentar sus capacidades físicas, mejorar su salud, aumentar las capacidades de entendimiento social, incrementar la seguridad y, además, mejorar la productividad y el crecimiento económico. La utilización de las NBIC está presente en diversos campos como la industria, la salud, la informática, energía, medio ambiente, problemáticas sociales, entre otros.

Los beneficios consecuentes de las tecnociencias objeto de estudio, se asocian primordialmente con el 
impulso de tecnologías eficientes y de bajo costo que podrán permitir acceso a buena parte de la población de países en desarrollo, a recursos vitales como el agua potable, la energía solar y el diagnóstico y tratamiento de algunas enfermedades. En otras palabras, podría ser el inicio de un cambio sustancial para minimizar la pobreza y para el impulso al desarrollo de las comunidades y países en vías de progreso y crecimiento económico.

Algunos ejemplos de aplicación de las NBIC como posibles curas al cáncer terminal, predicciones de problemas de salud, creación de componentes eficientes para productos y artefactos industriales y comerciales basados en la impresión 3D, estudio del cerero y las neuronas, análisis y manejo de grandes volúmenes de datos para la generación de información en tiempos muy cortos, entre otros, son esperanzadores y motivan a comprometerse e involucrarse activamente en el fomento, apoyo y uso de las tecnologías convergentes.

Los factores que favorecen la tendencia de utilización de las NBIC, están asociados con hechos que se han venido presentando en los últimos años y en la actualidad, tales como el tiempo y costo creciente en el desarrollo de medicamentos; la tendencia hacia la personalización de la medicina; la existencia de personal investigador entrenado en bioinformática, neuroinformática y nanomedicina; la madurez de la industria de dispositivos médicos; la existencia de diversos casos de éxito como los biochips; y la existencia de una cultura de alianzas entre empresas y de investigación cooperativa en el entorno académico.

No obstante, la competitividad y la búsqueda de respuestas y soluciones a problemas sociales, ambientales y de salud, constituyen otros factores que motivan la convergencia e integración de tecnologías. Cada día se irán desarrollando más aplicaciones de las NBIC en diversos campos como la industria, la salud, la informática, energía, ambiente, problemáticas sociales y otros, caracterizándose principalmente por que la ciencia no se define a partir de la especialización, sino más bien por la interdisciplinariedad de conocimientos en procura de la solución de problemas en común.

\section{Referencias}

Bueno, E. (2006). Las ciencias y tecnologías convergentes (NBIC): análisis de su papel en los parques científicos como espacios y agentes de I+D+i. Encuentros Multidisciplinares, 8(22), 64-74. Obtenido de https://dialnet.unirioja.es/servlet/articulo?codigo=1419306

Casalet, M. (2017). El paradigma de la convergencia del conocimiento: alternativa de trabajo colaborativo y multidisciplinario. México, D.F.: Flacso.

Foladori, G., \& Invernizzi, N. (2008). Las Nanotecnologías en América Latina. México, D.F.: Porrúa. https://doi.org/10.4067/S0718-65682009000200013

Martín, N. (2011). Sobre fullerenos, nanotubos de carbono y grafenos. (F. d. Madrid., Ed.) ARBOR(187), 115-131. https://doi.org/10.3989/arbor.2011.extran1117

Martín-Sánchez, F., \& López-Campos, G. (2006). Convergencia de tecnologías: nuevas oportunidades para el avance de la informática y de las ciencias biomédicas. Resvistaesalud.com, 2(6). Obtenido de https:/www.researchgate.net/publication/ 268347566_CONVERGENCIA_DE_TECNOLOGIAS_NUEVAS_OPORTUNIDADES_PARA_EL_AVANCE_DE_LA_INFORMATICA_Y_DE_ LAS_CIENCIAS_BIOMEDICAS 
Mejía, S. (1 de Marzo de 2019). La guatemalteca que creó una cura contra el cáncer terminal. Soy 502. Obtenido de https:/www.soy502.com/articulo/guatemalteca-descubre-cura-contra-cancer-taiwan-153

Rodríguez, J. (2009). Los usos sociales de la ciencia: tecnologías convergentes y democratización del conocimiento. Estudios Sociales. Revista de alimentación contemporánea y desarrollo regional, 17(34), 226-249. Obtenido de https://www.redalyc.org/articulo.oa?id=41711502009

Sáenz, T. (2006). Universidad para la Cooperación Internacional. Obtenido de http://www.ucipfg.com/ Repositorio/MIA/MIA-02/BLOQUE-ACADEMICO/Unidad\%201/ lectura\%20obligatoria/Tecnologias_convergentes.pdf

\section{Sobre el autor}

\section{Jorge Estuardo Morales}

Es Ingeniero Industrial por la Universidad de San Carlos de Guatemala, Magíster en Dirección y Gestión del Recurso Humano por la Universidad Mariano Gálvez de Guatemala, y actualmente es candidato a Doctor en Administración de Empresas y Capital Humano por la Universidad Galileo. Es docente universitario con más de 19 años de experiencia en el área profesional de la Ingeniería Industrial y en el área de métodos cuantitativos en posgrados de administración. Además se desempeña como facilitador y consultor empresarial e institucional gubernamental.

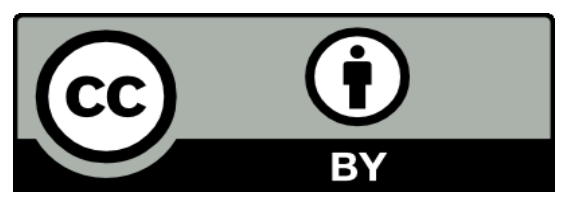

Este texto está protegido por una licencia CreativeCommons 4.0.

Usted es libre para compartir, copiar y redistribuir el material en cualquier medio o formato y adaptar el documento, remezclar, transformar y crear a partir del material para cualquier propósito, incluso comercialmente, siempre que cumpla la condición de atribución: usted debe reconocer el crédito de una obra de manera adecuada, proporcionar un enlace a la licencia, e indicar si se han realizado cambios. Puede hacerlo en cualquier forma razonable, pero no de forma tal que sugiera que tiene el apoyo del licenciante o lo recibe por el uso que hace. 